\title{
Trajectory of Change in Erectile Dysfunction Symptoms Following Coronary Artery Bypass Surgery in Malaysian Population
}

Nur Ayub $M A^{a}$, Suriati $M S^{b}$, Muhammad Ishamuddin $I^{a}$, Hairulfaizi $H^{a}$, Fahmi Pramaditto $A^{c}$, Mohamad Arif $M^{c}$, Mohd Ramzisham AR ${ }^{a}$, Mohd Rizal AM ${ }^{d}$, Katijjahbe MA ${ }^{\text {eff }}$

${ }^{a}$ Cardiothoracic Surgery, Heart and Lungs Center, University Kebangsaan Malaysia Medical Center

${ }^{b}$ Department of Psychiatry, Faculty of Medicine, University Kebangsaan Malaysia Medical Center

${ }^{c}$ Department of Surgery, Hospital Serdang

${ }^{d}$ Department of Community Health, Faculty of Medicine, University Kebangsaan Malaysia Medical Center

${ }^{e}$ Department of Physiotherapy, Hospital Canselor Tunku Mukhriz, University Kebangsaan Malaysia Medical Centre, 56000 Kuala Lumpur, Malaysia

${ }^{f}$ Department of Health Professions, Faculty of Health, Arts and Design, Swinburne University of Technology

\section{ABSTRACT}

\section{Keywords}

Coronary artery bypass surgery, Erectile Dysfunction (ED), International Index of Erectile Dysfunction

\section{Corresponding Author}

Dr. Nur Ayub Md Ali

Cardiothoracic Surgery, Heart and Lungs

Center, University Kebangsaan Malaysia

Medical Center

No Tel: 03-9145 6201

Email: drnurayub@gmail.com

Received: 13 July 2020; Accepted: 1 March 2021

Doi: https://doi.org/10.31436/imjm.v20i3
INTRODUCTION: Despite the advantages of Coronary artery bypass surgery (CABG), this procedure has been reported to have a significant impact on sexual activity. The study aims to assess the trajectory of change in erectile dysfunction (ED) symptoms following CABG. MATERIALS AND METHODS: 73 adult male participants were recruited. Participant primary data was measured using the International Index of Erectile Dysfunction (IIEF-5) at three-time points. RESULTS: Severe ED was most prevalent during 6 weeks following-operation (86.3\%); however, the proportion of severe ED symptoms reduced after 4 months following-operatively $(42.5 \%)$. There was a statistically significant difference in ED symptoms between pre-operatively and 6 weeks postoperatively $(\mathrm{p}<0.001)$. A significant improvement in the ED symptoms was demonstrated at 4 months $(p<0.001)$ compared to 6 weeks post-surgery, respectively. At 4 months, there was a marginally significant improvement in ED symptoms compared to baseline pre-operatively post CABG $(\mathrm{p}=0.064)$. Age significantly increases the chance in odds of having abnormal ED symptoms by $22 \%$ at 4 months postoperatively (aOR $=1.22,95 \%$ $\mathrm{CI}=1.06,1.41, \mathrm{p}<0.05)$. Other risk factors for abnormal ED symptoms following CABG, which include normal creatinine clearance, being a smoker, and having moderate to good LV function was not statistically significant. CONCLUSION: The present study has demonstrated a high prevalence of ED following CABG and the procedure caused a negative impact on the ED symptoms. However, there was a significant improvement in ED symptoms as the time from surgery increases and patients recovered. Further studies with larger sample size and longer recovery time are needed to assess the trajectory of recovery in ED symptoms in this population.

\section{INTRODUCTION}

Coronary artery bypass surgery (CABG) is considered the gold standard treatment for triple vessel disease and patients with multiple comorbidities., ${ }^{1,2}$ Despite the advantages of CABG, this procedure has been reported to have a significant impact on sexual activity. ${ }^{3,4}$ The origin of sexual dysfunction following cardiac surgery is associated with coronary artery disease (CAD). 5,6 Sexual dysfunction may be classified into four main groups: i) loss of desire (libido), ii) erectile dysfunction (ED), iii) ejaculatory insufficiency, and iv) anorgasmic states. ${ }^{3}$ Of those sexual dysfunction, ED has been reported to have a high incidence with the prevalence between 2-88\% with an overall age-standardized prevalence of $37 \%$ following established cardiovascular disease.6,8 $\mathrm{ED}$ is associated with significant morbidity, mortality and were reported to have negative impact on life psychologically resulting to stress, depression, loneliness and decreased quality of social relationship with subsequently reduce health-related quality of life. 5,8

IMJM Volume 20 No.3, July 2021 
Empirical evidence has established several predisposing factors that contribute to ED and this includes age, multiple comorbidities, smoking, sedentary lifestyle and depression. 5,8 Male sexual dysfunction affects about 10$25 \%$ of middle-aged and elderly men. ${ }^{9}$ A study conducted in Malaysia involving men over 40 years old who attended primary care clinic had shown about $69.5 \%$ of them reported having ED symptoms. ${ }^{10}$

Resumption of sexual activity is one of the important factors of psychosocial recovery after cardiac surgery. Studies have shown mixed results on the effect of CABG on ED.4,9,11 While the existence of ED in CAD patients and some of the aetiology such as endothelial damage and atherosclerosis are known, the effect of CABG surgery and its impact on $E D$ is controversial. ${ }^{11,12}$ There is relatively less evidence to support that CABG will improve ED symptoms. ${ }^{9,13}$ To date, there has been no study conducted regarding ED symptoms in men post-CABG in Malaysia. A prospective observational study was conducted to explore the prevalence and severity of ED symptoms in patients undergoing CABG, both pre- and postoperatively, which will provide valuable data on the natural history of ED symptoms post-CABG. Therefore, the aim of this study is to assess trajectory of change in ED symptoms in patients following coronary artery bypass surgery (CABG).

\section{METHODS AND METHODOLOGY}

The study was conducted and reported in accordance with the 'Strengthening the Reporting of Observational Studies in Epidemiology' (STROBE) guidelines. ${ }^{14}$ Participants were recruited prospectively from two tertiary hospitals in Kuala Lumpur, from June 2018 to June 2019, who underwent CABG.

Participants were eligible for the study if they met the following criteria: i) male with isolated CABG, ii) Malay and/or English-speaking, iii) able to provide informed consent, iv) adult less than 65 years old, and v) no ED drug used pre- and post-operatively. Participants were ineligible if they were high risk patients admitted to ICU with intra-aortic balloon pump (IABP) or without IABP (as determined by their respective cardiothoracic surgeons/team pre-operatively).
The study was approved by the Medical Research and Ethical Committee Board at National University of Malaysia (Reference Number: JEP 2013-103) and Board of Medical Research and Ethics Committee Ministry of Health Malaysia (Reference Number: NMRR-19-39745544). All patients for the two centres underwent the same method, standardized generalized anaesthesia and surgical techniques.

Participants were subjected to a structured interview using the International Index of Erectile Dysfunction (IIEF-5). This questionnaire is an abridged five-item version of the 15-item International Index of Erectile Function (IIEF). ${ }^{15,16}$ It was developed to diagnose the presence and severity of $\mathrm{ED}$. The score ranges from one (1) (severe symptom) to five (5) (no symptom). A total score of 25 with lower scores indicating worst function. It is a self-reported instrument administered face-to-face with high degree of sensitivity and specificity in measuring patients with ED symptoms. ${ }^{15,16}$ Participants were required to answer the same questionnaire at three different time points namely; i) pre-surgery, ii) 6 weeks post-surgery, and iii) four months post-surgery. The interview was performed with participants privately by the investigator or research assistant during patient admission in the ward and postoperatively in the outpatient clinic setting. For patients who were unable to attend the outpatient appointment, they were contacted for telephone interview. Other data such as patient information including phone number, patient clinical notes such as medical history, blood investigation, and operation notes were obtained from the electronic hospital information system.

The sample size was based upon the prevalence of ED symptoms following $\mathrm{CABG}$ of $83 \% .{ }^{4}$ A single proportion sample size calculation formula with finite population correction was used. ${ }^{17}$ Using finite population correction of $200 \mathrm{CABG}$ patients per year, a precision of 0.1 and confidence level of $95 \%, 73$ samples were needed after adjusting for a $20 \%$ drop-out rate.

Statistical analyses were performed using IBM SPSS version 23.0 for Windows. Data was assessed for normality using the Kolmogorov-Smirnov test. The numerical data such as age and clamping time was not 
normally distributed. Descriptive statistics using median (interquartile range) for continuous data and frequency (percentage) was used to summarize data, which included the prevalence of ED symptoms and participant demographics. Participants were then categorized based on IIEF score into those who has ED (IIEF score 5-21) and no ED (IIEF score 22-25). McNemar test was used to analyse the categorical data (has ED and no ED) over time.

Multivariate logistic regression was used to control confounders of demographic data, medical data, and pre-operative variables (independent variables) based on ED status (dependent variable) at 4 months postsurgery. For all tests, $p<0.05$ were considered to be statistically significant and confidence interval reported as necessary.

\section{RESULTS}

A total of 122 potential patients were assessed but only 73 adult male undergoing CABG were recruited for the study with the mean age of $54.6( \pm 6.9)$ years old. Eighty -nine percent $(n=61)$ of the participants had multiple comorbidities with more than $50 \%(n=42)$ having good left-ventricular (LV) function. Data was analysed for 73 patients, only 2 patients had 1 time point data missing for the IIEF-5 score. This was taken into consideration as part of statistical analysis. The difference was not significant. Table 1 demonstrates the demographic and clinical characteristics of participants.

Changes in sequential ED severity symptoms from preoperative, 6 weeks and 4 months post-surgery using IIEF-5 are shown in Table 2. Severe ED was most prevalent at 6 weeks post-surgery amongst $82.2 \%$ of the participants. However, the proportion of severe ED symptoms reduced after 4 months post-surgery with only $42.5 \%$ of the study participants.

The number of participants with no ED and has ED symptoms pre-surgery were compared to with those at 6 weeks post-surgery and 4 months post-surgery are described in Table 3. There was significant difference in the ED symptoms pre-operatively and 6 weeks postCABG ( $p<0.001)$. ED symptoms improved significantly between 6 weeks post-surgery and 4 months post-
Table 1: Demographic and clinical characteristic of study sample $(\mathrm{n}=73)$

\begin{tabular}{cl}
\hline Characteristics & $\mathbf{n}(\%)$ \\
\hline Age(years), mean (SD) & $54.63 \pm 6.86$ \\
Smoking History & \\
No/never smoke & $35(53.0)$ \\
Current smoker & $27(40.9)$ \\
Ex-smoker & $4(6.1)$ \\
NYHA* & \\
I & $33(45.2)$ \\
II & $26(35.6)$ \\
III & $6.8(7.8)$ \\
Left ventricular function & \\
Good & $42(57.5)$ \\
Moderate & $18(24.7)$ \\
Poor & $7(9.6)$ \\
Very poor & $1(1.4)$ \\
Co-morbidities & \\
Single & $7(10.3)$ \\
Multiple & $61(89.7)$ \\
No ED & \\
Mild ED & $30(41.1)$ \\
Mild-moderate ED & $8(11.0)$ \\
Moderate ED & $13(17.8)$ \\
Severe ED & $13(17.8)$ \\
IIEF-5 score category & $9(12.3)$ \\
\hline
\end{tabular}

$*$ NYHA $=$ New York Heart Association Functional Classification

CABG $(p<0.001)$. After four months of post-CABG, there was no significant difference in ED symptoms compared to pre-operative ED symptoms $(p=0.064)$.

Table 2: Distribution of erectile dysfunction symptoms based on IIEF-5 according to time-point $(\mathrm{n}=73)$

\begin{tabular}{lll}
\hline $\begin{array}{l}\text { IIEF-5 classification according } \\
\text { to time point }\end{array}$ & $\mathbf{n}$ & $\mathbf{( \% )}$ \\
\hline Pre-operative & & 12.3 \\
Severe ED & 9 & 17.8 \\
Moderate ED & 13 & 17.8 \\
Mild-Mod ED & 13 & 11.0 \\
Mild ED & 8 & 41.1 \\
No ED & 30 & \\
6 weeks following-operation & & 82.2 \\
Severe ED & 60 & 5.5 \\
Moderate ED & 4 & 1.4 \\
Mild-Mod ED & 1 & 1.4 \\
Mild ED & 1 & 5.5 \\
No ED & 4 & \\
4 months following-operation & & 42.5 \\
Severe ED & 31 & 15.1 \\
Moderate ED & 11 & 5.5 \\
Mild-Mod ED & 4 & 9.6 \\
Mild ED & 7 & 26.0 \\
No ED & 19 &
\end{tabular}


Table 3: Trajectory of change in erectile dysfunction symptoms following coronary artery bypass surgery (CABG) comparing at 3 time points.

\begin{tabular}{lcclccc}
\hline & \multicolumn{3}{c}{ 6 weeks } & \multicolumn{3}{c}{4 months } \\
\cline { 2 - 7 } Pre- & No & With & p value & No & With & p value \\
operative & ED & ED & & ED & ED & \\
& (n) & (n) & & (n) & (n) & \\
& 3 & 23 & $<0.001^{* *}$ & 13 & 14 & 0.064
\end{tabular}

(n)

$\begin{array}{lllll}\text { With ED\# } & 1 & 44 & 5 & 41\end{array}$

(n)

${ }_{n}=$ number, no erectile dysfunction (IIEF-5 score 22-25). \#with erectile dysfunction (IIEF score 5-21)

McNemar Test, **significant at $p<0.05$

We found that the older the patient, there was a significant $22 \%$ incremental change in having worsening ED symptoms 4 months following-CABG (aOR=1.22, $95 \% \mathrm{CI}=1.06,1.41, \mathrm{p}<0.05) \quad($ Table 4$)$. The other independent variables such as clamping time, premorbid factors, usage of betablocker, creatinine clearance, smoking status, NYHA and left ventricular function were not significant predictors of ED symptoms based on IIEF-5 scores.

Table 4: Predictor of worsening erectile dysfunction based on IIEF-5 score 4 months post-coronary artery bypass $(\mathrm{CABG})$ surgery using multivariate logistic regression

\begin{tabular}{|c|c|c|c|c|}
\hline \multirow{2}{*}{$\begin{array}{l}\text { Independent } \\
\text { variable }\end{array}$} & \multirow{2}{*}{$\begin{array}{l}\text { Adjusted } \\
\text { odds ratio } \\
(\mathrm{aOR})\end{array}$} & \multicolumn{2}{|c|}{$\begin{array}{l}95 \% \text { Confidence } \\
\text { Interval }\end{array}$} & \multirow{2}{*}{$p$ value } \\
\hline & & $\begin{array}{l}\text { Lower } \\
\text { limit }\end{array}$ & $\begin{array}{l}\text { Upper } \\
\text { limit }\end{array}$ & \\
\hline Age & 1.22 & 1.06 & 1.41 & $0.006^{*}$ \\
\hline Clamping time & 0.994 & 0.98 & 1.01 & 0.43 \\
\hline
\end{tabular}

Premorbid

$\begin{array}{lllll}\text { Multiple } & 0.86 & 0.07 & 10.72 & 0.91 \\ \text { Single } & 1 & & & \end{array}$

Beta-blocker

$\begin{array}{lllll}\text { Yes } & 0.997 & 0.13 & 7.87 & 0.998 \\ \text { No } & 1 & & & \end{array}$

Creatinine

clearance

$\begin{array}{lllll}\text { Normal } & 1.87 & 0.32 & 10.77 & 0.49 \\ \text { Impaired } & 1 & & & \end{array}$

Smoking status

Yes $\quad 3.27$

No 1

NYHA

$\begin{array}{lllll}\begin{array}{l}\text { Class II } \\ \text { and III }\end{array} & 0.58 & 0.11 & 3.09 & 0.52 \\ \text { Class I } & 1 & & \\ \begin{array}{l}\text { Ventricular } \\ \text { tion }\end{array} & & & & \\ \begin{array}{l}\text { Moderate } \\ \text { to good }\end{array} & 3.05 & 0.31 & 30.15 & 0.34 \\ \begin{array}{l}\text { Very poor } \\ \text { to poor }\end{array} & 1 & & & \\ \end{array}$

*significant at $\mathrm{p}<0.05$

IMJM Volume 20 No.3, July 2021

\section{DISCUSSION}

Our findings showed a high prevalence of ED following $\mathrm{CABG}$ and the procedure can caused negative impact on the ED symptoms. There was a significant worsening of ED symptoms at 6 weeks post-CABG. However, significant improvement of ED symptoms was found at 4 months compared to 6 weeks, and no significant difference in improvement compared to preoperative CABG as time from surgery increases and ED symptoms return to baseline. To the best of our knowledge, this is the first study to address a gap in the literature by providing additional detail pertaining to changes in erectile dysfunction symptoms in patients following elective CABG in Malaysia.

At 6 weeks post-CABG, 86\% of our participants reported severe degree of ED symptoms based on the IIEF-5 score. This is higher than previous studies within the CABG cohort ranging from $20.1-76.4 \% .3,9,13$ This may be due to the outcome measured used in this study were obtained at earlier post-operative periods compared to other studies.3,9,13,18 At 6 week time point, we postulated that the high incidence of ED were attributed to the acute alterations in circulatory homeostasis characterized by lower plasma testosterone post-CABG compared to pre-operative plasma testosterone concentration. $3,9,18$ Canbaz et al, 2002 reported that the level of testosterone was decreased significantly one day after $\mathrm{CPB}$ but returned to pre-operatively levels seven days post-CABG. However, the level of testosterone was still significantly lower compared with the pre-operative value. ${ }^{19}$ The time-point in our study may explain the high prevalence of severe ED symptoms and CABG procedure is an independent predictor of sexual dysfunction consistent with other studies. $3,9,13$

We also found significant improvement in ED symptoms 4 months post-CABG compared to 6 weeks post-CABG as this allowed patients to recover postsurgery. Our results concurred with a study that shows a marginal improvement in severe ED post-CABG (from $11.97 \%-8.21 \%$ ), and the surgery had a positive effect on ED symptoms 6 months post-CABG. ${ }^{4}$ This is attributed to higher tissue perfusion and improvement of penile erectile function after longer recovery time postCABG at this time point. ${ }^{4}$ We hypothesized that the 
longer recovery period after surgery may be a significant factor for future consideration in order to assess the trajectory of change in ED symptoms post-CABG.

Consistent with other studies, ${ }^{3-5,9,13}$ we found that age is the main risk factor which contributed to the prevalence of ED symptoms post-CABG in our study population. One study reported that increasing age was independent factor associated with a higher prevalence of ED in the elderly $(90.8 \%)$, followed by middle-aged $(83.3 \%)$ and young (73.3\%) men in Malaysia. ${ }^{20} \mathrm{~A}$ possible cause was the gradual decrease of physiological function as one's age progresses. ${ }^{20}$

Other risk factors such as creatinine clearance, being a smoker, clamping time, multiple comorbidities, usage of beta blocker and NYHA classification were not statistically significant. This may be due to small sample size which has reduced the chance of detecting a true effect. Interestingly, smoking did not appear as a predictor of ED as reported in our study. This may be due to our study population were younger where vascular risk factors such penile arterial blood flow was least likely to occur in the smoking group. ${ }^{21}$ Similarly, betablockers did not influence ED symptoms in our study. Despite the common usage of this drug in most of our patients, the impact of CAD and other underlying comorbidities maybe significant factors on ED symptoms other than betablockers. ${ }^{13}$ This finding is consistent with other studies on the use of betablockers which has no association with higher incidence of ED symptoms. ${ }^{13,22}$ These findings indicate that there may be underlying factors involved in changes of ED symptoms in addition to the $\mathrm{CABG}$ procedure. ${ }^{12}$

It is important to note that the cause of $\mathrm{ED}$ is multifactorial including psychological factors such as depression, anxiety and stress may be potential factors interfering with quality-of-life following CABG.23,24 Sexually assessment and counselling are part of recommendation in management of $\mathrm{ED}, 25,26$ and yet it is an overlooked aspect of cardiac rehabilitation after CABG surgery. We recommend that health professionals address sexual issues with their patients, ideally between 6 weeks to 4 months post-CABG with involvement of the patients' partners with the broader aim of addressing psychological wellbeing. 25,26
Further study is needed to assess the trajectory of change of ED symptoms in order to deliver the right timing for drug intervention and rehabilitation in the treatment of erectile dysfunction following CABG.

The limitation of this study is the small sample size and potential recall bias from patients answering the questionnaire. Small sample size reduces chance of detecting a true effect and recall bias that can increase measurement errors. Despite that, we have two missing data at one time-point each, and there is no drop-out case with the prevalent of ED symptoms that was similar to other studies. Therefore, we conclude that this possible bias did not change the results significantly.

\section{CONCLUSION}

The present study has demonstrated a high prevalence of ED following CABG. CABG procedure caused negative impact on the ED symptoms that there may be underlying factors involved in trajectory of changes of ED symptoms following CABG. However, there was a significant improvement of ED symptoms as time from surgery progresses and patients recovered further. We recommend information regarding sexual activity following $C A B G$ should be informed prior to surgery and integrated into post-surgery cardiac rehabilitation in order to reduce fear of having sexual activity after CABG. Further study with larger sample size and longer recovery time are needed to assess the trajectory of recovery in ED symptoms in this population.

\section{REFERENCES}

1. Deb S WH, Ko DT, Tsubota H, Hill S, Fremes SE. Coronary artery bypass graft surgery vs percutaneous interventions in coronary revascularization: a systematic review. JAMA. 2013;310(19):2086-95. .

2. Feldman TE, Brand, M. Van Den, Bass, E. J., Dyck, N. Van, Leadley, K., Dawkins, K. D., Mohr, F. W., et al. Percutaneous Coronary Intervention versus Coronary-Artery Bypass Grafting for Severe Coronary Artery Disease. N Engl J Med. 2009;360:961-72. 
3. Forouzannia SK AM, Hekmatimoghaddam S, Hassan Sayegh SA. Sex hormone levels and sexual dysfunction in men after coronary artery bypass graft. Iran J Reprod Med. 2012;10(5):425-8.

4. Ziabakhsh Tabary S, Mokhtari-Esbuie, F. \& Fazli, M. Evaluations of erectile dysfunction before and after on-pump coronary artery bypass graft surgery. . Caspian Journal of Internal Medicine. 2014;5(4):209-12.

5. Feldman HA GI, Hatzichristou DG, Krane RJ, McKinlay JB. . Impotence and its medical and psychosocial correlates: results of the Massachusetts Male Aging Study. . J Urol 1994;151: . 1994;151:54-61.

6. Park K HE, Kim SO. . Prevalence and medical management of erectile dysfunction in Asia. Asian J Androl. 2011;13(4):543-9.

7. Cheng JY NE, Chen RY, Ko JS. Prevalence of erectile dysfunction in Asian populations: a metaanalysis. Prevalence of erectile dysfunction in Asian populations: a meta-analysis. Int J Impot Res. 2007;19:229-44.

8. Tan HM LW, Ng CJ, Chen KK, Sugita M, Ishii N, Marumo K, Lee SW, Fisher W, Sand M.

Prevalence and correlates of erectile dysfunction (ED) and treatment seeking for ED in Asian Men: the Asian Men's Attitudes to Life Events and Sexuality (MALES) study. J Sex Med. 2007;4 (6):1582-92.

9. Foruzan-Nia SK, Abdollahi, M. H., Hekmatimoghaddam, S. H., Namayandeh, S. M. \& Mortazavi, M. H. . Incidence of sexual dysfunction in men after cardiac surgery in Afshar hospital, Yazd. Journal of Computational and Theoretical Nanoscience. 2011;9(2):89-94.

10. Ab Rahman AA, Al-Sadat, N. \& Yun Low, W. Prevalence of erectile dysfunction in primary care setting, Malaysia. Journal of Men's Health 8. 2011;8 (SUPPL. 1):S50-S3.

11. Akbulut B, Uçar HI, Öç B, Öç M, Karabay C, Özyüksel A, Farsak B, . Erectile function after coronary artery bypass surgery. Anatolian Journal of Clinical Investigation. 2008;2(4):146-9.

12. Pournaghash-Tehrani S, Etemadi, S. . ED and quality of life in CABG patients: an intervention study using PRECEDE-PROCEED educational program. . Int J Impot Res. 2014;26:16-9.
13. Schumann JZM, Di Valentino M, Piazzalonga S and Hoffmann A. Sexual Dysfunction before and after Cardiac Rehabilitation. Rehabilitation

Research and Practice. 2010:1-8.

14. von Elm EAD, Egger M, et al. STROBE Initiative. The Strengthening the Reporting of Observational Studies in Epidemiology (STROBE) statement: guidelines for reporting observational studies. J Clin Epidemiol 2008;61 (4):344-49.

15. Rosen Rc AS, Giuliano F. Research instruments for the diagnosis and treatment of patients with erectile dysfunction. Urology. 2006;68:16-9.

16. Tang Z, Li D, Zhang X, Yi L, Zhu X, Zeng X, Tang Y. Comparison of the simplified International Index of Erectile Function (IIEF-5) in patients of erectile dysfunction with different pathophysiologies. BMC Urology. 2014;14(1).

17. Thrusfield MVE, 2nd Edition, Blackwell Science, Oxford, UK (p 183). (n.d.). https:// epitools.ausvet.io/oneproportion.

18. Heaton JPEH, Adams MN, Smith KMA. Coronary artery bypass graft surgery and it's impact on erectile dysfunction; a preliminary retrospective study. Int J Impot Res. 1996;8:35-9.

19. Canbaz SET, Sunar H, Cikirikcioglu M, Acipayam M, Duran E. The effects of cardiopulmonary bypass on androgen hormones in coronary artery bypass surgery. . J Int Med Res. 2002;30:9-14.

20. Nordin RST, Kaur A, Loh KP, Miranda S, Prevalence and predictors of erectile dysfunction in adult male outpatient clinic attendees in Johor, Malaysia. Singapore Med J 2019; 60(1): 40-47. 2019;60(1):40-7.

21. Kendirci MNS, Hellstrom WJG. The impact of vascular risk factors on erectile function. Drugs of Today. 2005;41(1):65-74.

22. Maroto-Montero JM, Portuondo-Maseda MT, Lozano-Suárez M, Allona A, de Pablo-Zarzosa C, Morales-Durán MD, Muriel-Garcia A, RoyuelaVicente A. Erectile dysfunction in patients in a cardiac rehabilitation program. Rev Esp Cardiol. 2008;61(9):917-22.

23. Pournaghash-Tehrani S, Etemadi S. ED and quality of life in CABG patients: an intervention study using PRECEDE-PROCEED educational program. Int J Impot Res, 2014. 26:16-19. 
24. Laumann EO, P.A., Rosen RC . Sexual dysfunction in the United States: prevalence and predictors. JAMA, 1999. 281: 537-544.

25. Steinke EE, et al., Sexual counselling for individuals with cardiovascular disease and their partners: A Consensus Document From the American Heart Association and the ESC Council on Cardiovascular Nursing and Allied Professions (CCNAP). European Heart Journal, 2013. 34(41): 3217-3235.

26. Levine, G.N., et al., Sexual activity and cardiovascular disease: a scientific statement from the American Heart Association. Circulation, 2012. 125(8):1058-72. 